\title{
Individual monitoring of gonad development in the European flat oyster Ostrea edulis by in vivo magnetic resonance imaging
}

\author{
Armel Davenel ${ }^{a, c, ~}{ }^{*}$, Ricardo González ${ }^{b}$, Marc Suquet ${ }^{b}$, Stéphane Quellec ${ }^{a, c}$ and René Robert ${ }^{b}$ \\ ${ }^{a}$ Cemagref, UR TERE, 17 avenue de Cucillé-CS 64427, F-35044 Rennes, France \\ ${ }^{b}$ Ifremer, UMR100, LPI, Station Expérimentale d'Argenton, 29840 Argenton en Landunvez, France \\ ${ }^{\mathrm{c}}$ Université Européenne de Bretagne, France \\ *: Corresponding author : A. Davenel, Tel.: + 3322348 2160; fax: + 3322348 2115., email address : \\ armel.davenel@cemagref.fr
}

\begin{abstract}
:
Previous studies have shown that magnetic resonance imaging is a very appropriate non-invasive technique for quantifying the growth of somatic and gonadic tissues and sex determination in the Pacific oyster, Crassostrea gigas. Despite a thinner gonad, harder to distinguish than that of the Pacific oyster, we showed in this study that it was possible to distinguish and quantify the development of the gonad of the European flat oyster, Ostrea edulis, using a 3D MRI sequence with a suitable finer resolution. Compared to T2-weighted images, theoretically the most appropriate for good anatomical description, T1-weighted images were more suited to gonad observation. The gonad development was quantified by the number of voxels with higher grey level. Larvae were then depicted in the intervalvar cavity of spawning females. MRI imaging is a non-invasive method that is well suited to the description of gametogenesis in the European flat oyster.
\end{abstract}

Keywords: Oyster; Ostrea edulis; Gonad development; Magnetic resonance imaging 


\section{Introduction}

The European flat oyster, Ostrea edulis, is a native European species and traditionally is an attractive product for consumption in a range of European countries. This species is highly valued and sought after on the European seafood market. However, supplies are limited because of declines in fisheries due to recruitment failure and the occurrence of diseases caused mainly by parasites and environmental stress.

Aquaculture may counteract decline in availability and, in term of biodiversity, it is also important to sustain the production of healthy and genetically differentiated populations along European coasts. Spat production of flat oysters has been a challenge over the years compared to the more easily cultured introduced species such as Pacific oyster, Crassostrea gigas. Research focused on the flat oyster is therefore essential to maintain large enough production to meet demands and retain the market share. However, the ecology and physiology of this bivalve are not fully understood, and consequently monitoring of growth and reproduction, both in the field and in hatcheries, is still based on empirical factors.

Investigation of soft tissues in bivalves classically relied on the removal of the hermetic shell that protects the animal. Anatomical structures can then be studied by means of histological sections (Chavez-Villalba et al., 2002; Dridi et al., 2007). Gametogenesis can be quantitatively assessed using image analysis (Fabioux et al., 2005). This has provided much valuable information, but has two major disadvantages: theses methods are time consuming and necessarily destructive, preventing successive samplings on the same individuals. That is why, practical anaesthesia and sampling protocols have been developed in some bivalves such as Pacific oyster (Namba et al., 1995; Suquet et al., 2009), enabling sex determination and maturation stage observation without sacrificing animals. However, the assessment of biometric parameters (gonad wet weight and total body weight) facilitating the determination of condition index, is prevented. Furthermorer, analysis of physiological and biochemical changes requiring large volume tissue samples still involves the sacrifice of numerous specimens and the preparation and analysis of several samples.

Non-invasive and quantitative procedures have therefore been developed and have proved promising after preliminary trials (Pouvreau et al., 2006). Non-invasive characterization of gonad maturation and sex determination of the Pacific oyster by Magnetic Resonance Imaging (MRI) has already been successfully applied using longitudinal relaxation T1-weighted MRI sequences (Davenel et al., 2006; Hatt et al., 2009). MRI is the most appropriate technique for quantification of the growth of somatic and gonadic tissues because this method is non-invasive and successive sampling may be performed on the same individuals, thus facilitating descriptive studies such as gametogenesis.

We report here the ability of MRI to monitor gonad development in individual European flat oysters, Ostrea edulis, until spawning. The European flat oyster is a larviparous species, the larvae of which are released after 7 to 10 days of development. Compared to the Pacific oyster, the fecundity of the European flat oyster is lower (1 to 2 million eggs; Walne, 1964), and smaller gonad size is reported. Any MRI protocol must therefore take into account these reproduction features.

\section{Materials and methods}

\subsection{Origin of animals and preparation}

Three hundred flat oysters (4 years of age, Bonamia and Marteilia free), originating from Norway, were transferred to the Argenton experimental hatchery (West Brittany). Oysters were maintained from April to August 2009 , in $700 \mathrm{~L}$ flow-through tanks at $19^{\circ} \mathrm{C}$ with permanent light, and continuously fed according to Chávez-Villalba et al. (2002). .

Twelve non-anaesthetised oysters were brought $250 \mathrm{~km}$ to Cemagref in Rennes (Brittany, France) on five dates (April 7, April 29, May 13, June 2, July 10, 2009) for MRI investigations 
without any specific anaesthetization procedure. Before MRI measurements, animals were soaked in sea water to expel any air bubbles which can cause artefacts on images. Sea water was maintained at room temperature and shell opening was facilitated by the addition of phytoplankton. After expelling air bubbles, oyster shells were maintained closed with a rubber band during MRI scanning.

\subsection{MRI measurements}

In this new study, NMR measurements were performed on the European flat oyster at Cemagref (Rennes, Brittany, France) with a Siemens Avento imager operating at $1.5 \mathrm{~T}(60 \mathrm{MHz})$ equipped with a "knee" probe which allowed the simultaneous investigation of two oysters. The stronger magnetic field of this machine allowed us to obtain more precise images with higher spatial resolution and to monitor the development of the very thin gonad observed in individual flat oysters. Each oyster was scanned using two high resolution 3D MRI protocols (Table 1). A T2 weighted Turbo 3D Spin Echo sequence provided 48 contiguous images, highlighting water in the shell and chambers and pericardial cavities (Fig. 1a). A T1-weighted Turbo Gradient Echo sequence provided 52 contiguous images, highlighting animal tissues and particularly gonadic tissues (Fig. 1b).

\subsection{Image analysis}

Examination of raw images obtained from an MRI scanner can lead to erroneous interpretations of variations in the grey level in the images: several sources of spatial inhomogeneities in the apparatus related to the inhomogeneity of the magnetic field and the geometry of the transmitting and receiving probes lead to undesirable variations in intensity in the images. Using homogeneous doped water phantoms as reference, we calculated that the grey levels of the oyster placed in the lower part of the MRI probe were $7 \%$ lower than the grey levels of the oyster placed in the upper part. The grey levels of the image, originating from the lower part of the raw images, were therefore multiplied by 1.07 after automated segmentation of the raw images in two under-images to separate each of the two oysters, to obtain comparable grey levels for all the oysters scanned.

Although it was possible to obtain a reliable assessment of the development of somatic and gonadic tissues by visualizing the images, we tried to reduce the influence of the human input by exploiting the overall histograms of voxels in all the images and setting thresholds to separate tissues, while counting the somatic index reflecting the volume occupied by flesh and the gonad index reflecting the volume occupied by the gonad.

\section{Results}

\subsection{Choice of MRI sequence to monitor gonad development and tissue evolution}

Although the T2-weighted images (Fig. 1a) offered higher resolution and better signal to noise ratio and were theoretically the most appropriate for accurate anatomical description, the best contrasts for the characterization and segmentation of the gonad were obtained with T1weighted images (Fig. 1b). In particular, the highlighting of the gonad before spawning was very significant, while the gonad gave a low signal that was difficult to differentiate from the signal of the background and the signal of the shell in T2-weighted images. Therefore, in addition to visual observation and the method successfully used previously to segment MRI T1-weighted images of the Pacific oyster, we sought to characterize the gonad development of each oyster with a gonad index (the number of voxels of grey level $>150$ ) and the flesh occupation by a somatic index (the number of voxels of grey level $>90$ ) to eliminate background, shell and seawater in images (Fig. 2). 


\subsection{Monitoring of gonad development and detection of spawning of gametes}

Four of the twelve oysters died during the experiments: oyster 9 died after April 29, oyster 7 after May 13 and oysters 5 and 6 after June 2. The most representative T1-weighted images of the gonads of 10 of the 12 oysters scanned at the five dates is reported in Figure 3, including oysters 5 and 6 . Female gametes liberated in the intervalvar cavity, and probably fertilized by externally released sperm, were clearly visible for oysters 1, 2, 4 and 11 at different dates: larvae appeared as a grey volume in the gills. At the date preceding this stage when larvae were detected, the grey level of pixels corresponding to the gonad was very high and clearly visible in the images. We were therefore also able to determine the stage preceding spawning for oysters 5, 8 and 12, despite the fact that one corresponding animal died thereafter (oyster 5 ), or the larvae were not visible after their expulsion into the surroundings following an incubation period in the branchial chamber of 8-10 days, and probably because the time interval between the two successive samples was too long for these individual oysters. No gonad development was discernible for oysters 3, 6 and 10.

It was possible to estimate the gonad development by counting the number of voxels at grey level > 150 (Fig. 4a and 4b). The number reached 5000 to 6200 voxels for oyster 1, 2, 8, 11 and 12 just before spawning, i.e. a gonad volume between 1.10 and $1.37 \mathrm{~cm}^{3}$. Volume was only significantly lower $\left(0.44 \mathrm{~cm}^{3}\right)$ for oyster 5 just before death.

By counting voxels at grey levels $>90$ (Fig. 4c and 4d), it was possible to propose a raw somatic index. The index clearly increased from April to July for oysters 3 and 10 (Fig. 4d) but these oysters showed no apparent development of the gonad during this period. It was the same for oysters 2 and 4 after spawning, the stage at which this index was the lowest. The index did not vary significantly during the period of gonad maturation before spawning.

\section{Discussion}

This new study confirms the potential of nuclear magnetic resonance in the non-invasive phenotype characterization of bivalves. Because of the development of a thick gonad, we have previously shown the possibility of monitoring and measuring the development of the gonad in the Pacific oyster with a low-field $0.2 \mathrm{~T}$ MRI imager and with medium spatial resolution $\left(0.94^{\star} 0.94 * 4 \mathrm{~mm}^{3}\right)$ (Davenel et al., 2006). In this study tracking individual European flat oysters, we individually monitored a dozen oysters between April and July 2009 during gonad maturation. Despite the development of a thinner gonad, using a higher field (1.5T) with better spatial resolution $\left(0.47^{\star} 0.47^{\star} 1 \mathrm{~mm}^{3}\right)$ we were able to monitor and quantify gonad development until spawning.

We confirmed that T1-weighted MRI sequences are particularly well suited to highlight the gonad of the European flat oyster. It was possible during monitoring to visualize the incubation of larvae in the intervalvar cavity, providing evidence that the gonad observed days earlier belonged to a female animal. Our previous studies on the Pacific oyster showed that the female gonad provided a clearer signal that the male gonad. The gonad index proposed to monitor the development of the gonads just before spawning and visual observation showed that seven of the ten oysters presented in Fig. 3 would clearly develop a female gonad during the observation period. The three others did not seem to have developed a gonad, unless it was a male gonad with a weaker signal than that of a female gonad and little different from that of the viscera. This hypothesis could not be confirmed during this study because we did not want to sacrifice any animal.

The somatic index adopted in this study, currently validated to reflect only the weight of flesh in the Pacific oyster (Davenel et al., 2006), was lowest when the animal had just spawned. In the following period, it was more often growing progressively and then seemed to slow down during the gonad development phase. While the female gonad provided a clearer signal during this phase, the signal from the viscera tended to decrease. It is possible that there is accumulation of reserves before the gonad development phase and transfer of a proportion of the reserves accumulated in the organs to the gonad during gonad development. We have previously demonstrated that the somatic index proposed here in the case of the Pacific oyster (based on 
the number of voxels with grey levels higher than 90) is well correlated with flesh weight and thus mainly reflects the volume occupied by the flesh (Davenel et al, 2006). The grey level intensity in T1-weighted MRI images is highly correlated with dry matter content of tissues and particularly increased by the presence of lipids.

In conclusion, non-invasive imaging using MRI provides very valuable information for the individual monitoring of gonad development in the European flat oyster. Further studies, supplemented by biopsy and dissection, should be undertaken to characterize the development of the male gonad and to develop a new somatic index more suited to reflecting the accumulation of reserves including lipids. This would improve the existing relationship between the grey level in MRI image and dry matter content of the different tissues of the oyster.

\section{Acknowledgements}

The research leading to these results has received funding from the European Community's Seventh Framework Programme FRP/2007-2013 under grant agreement n²22043 (Settle project).

\section{References}

Chávez-Villalba, J., Pommier, J., Andriamiseza, J., Pouvreau, S., Barret, J., Cochard, J.C., Le Pennec, M., 2002. Broodstock conditioning of the oyster Crassostrea gigas: origin and temperature effect. Aquaculture 214 (1-4), 115-130.

Davenel, A., Quellec, S., Pouvreau, S., 2006. Non invasive characterization of gonad maturation and determination of the sex of Pacific oysters by MRI. Magn. Reson. Imaging 24 (8), 1103-1110.

Dridi, S., Salah Romdhane, M., Elcafsi, M., 2007. Seasonal variation in weight and biochemical composition of the Pacific oyster, Crassostrea gigas in relation to the gametogenic cycle and environmental conditions of the Bizert lagoon, Tunisia. Aquaculture 263 (1-4), 238-248.

Fabioux, C., Huvet, A., Le Souchu, P., Le Pennec, M., Pouvreau, S., 2005. Temperature and photoperiod drive Crassostrea gigas reproductive internal clock. Aquaculture 250 (1-2), 458470.

Hatt, P.J, Davenel, A., Pierre-Antoine Eliat, P.A, Quellec, S., 2009. Magnetic resonance imaging as a means to assess the body growth and the gonad development of the oyster Crassostrea gigas. Aquat. Living Resour. 22, 331-339.

Namba K., Kobayashi M., Aida S., Uematsu K., Yoshida M., Kondo Y., Miyata Y., 1995. Persistent relaxation of the adductor muscle of oyster Crassostrea gigas induced by magnesium ion. Fish. Sci. 61, 241-244.

Pouvreau, S., Rambeau, M., Cochard, J.C., Robert, R., 2006. Investigation of marine bivalves morphology by in vivo MR imaging: first anatomical results of a promising technique. Aquaculture 259 (1-4), 415-423.

Suquet, M., De Kermoysan, G., Arya, R.G., Queau, I., Lebrun, L., Le Souchu, P., Mingant, C., 2009. Anesthesia in Pacific oyster, Crassostrea gigas. Aquat. Living Resour. 22 (1), 29-34.

Walne, P.P.R., 1964. Observations on the fertility of the oyster Ostrea edulis. J. Mar. Biol. Assoc. U.K. 44: 293-310. 
Tables

\begin{tabular}{|l|l|l|}
\hline MRI sequence & T2-weighted 3D turbo spin echo & T1-weighted spoiled gradient echo \\
\hline Slice number & 48 & 52 \\
\hline Slice thickness $(\mathrm{mm})$ & 1 & 1 \\
\hline FOV $(\mathrm{mm} \times \mathrm{mm})$ & $75 \times 100$ & $75 \times 120$ \\
\hline Matrix & $192 \times 256$ & $160 \times 256$ \\
\hline Pixel size $(\mathrm{mm} \times \mathrm{mm})$ & $0.39 \times 0.39$ & $0.47 \times 0.47$ \\
\hline TR $(\mathrm{ms})$ & 2000 & 11 \\
\hline TE $(\mathrm{ms})$ & 150 & 4.76 \\
\hline Flip angle $\left({ }^{\circ}\right)$ & 170 & 20 \\
\hline Bandwidth $(\mathrm{Hz} / \mathrm{pixel})$ & 168 & 200 \\
\hline Excitation number & 1 & 6 \\
\hline Acquisition time & 13 min & $8 \mathrm{~min}$ \\
\hline
\end{tabular}

Table 1. Parameters of the two 3D MRI sequences used to describe and quantify flesh and gonad development.

\section{Figures}

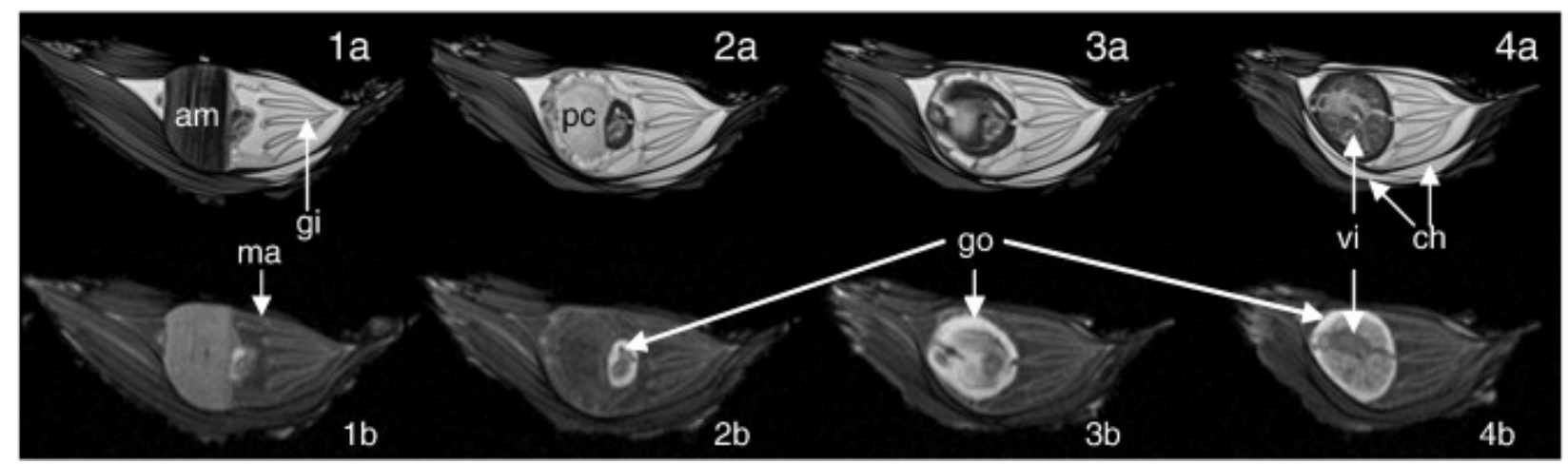

Fig. 1. The same four most representative transverse images through the body of flat oysters Ostrea edulis from the 48 images of the T2-weighted Turbo 3D Spin Echo sequence (a), and the 52 images of the T1-weighted Gradient Echo sequence (b). Main organs identified: adductor muscle (am), viscera (vi), gills (gi), gonad (go), pericardial cavity (pc), mantle (ma), shell chamber (ch). 


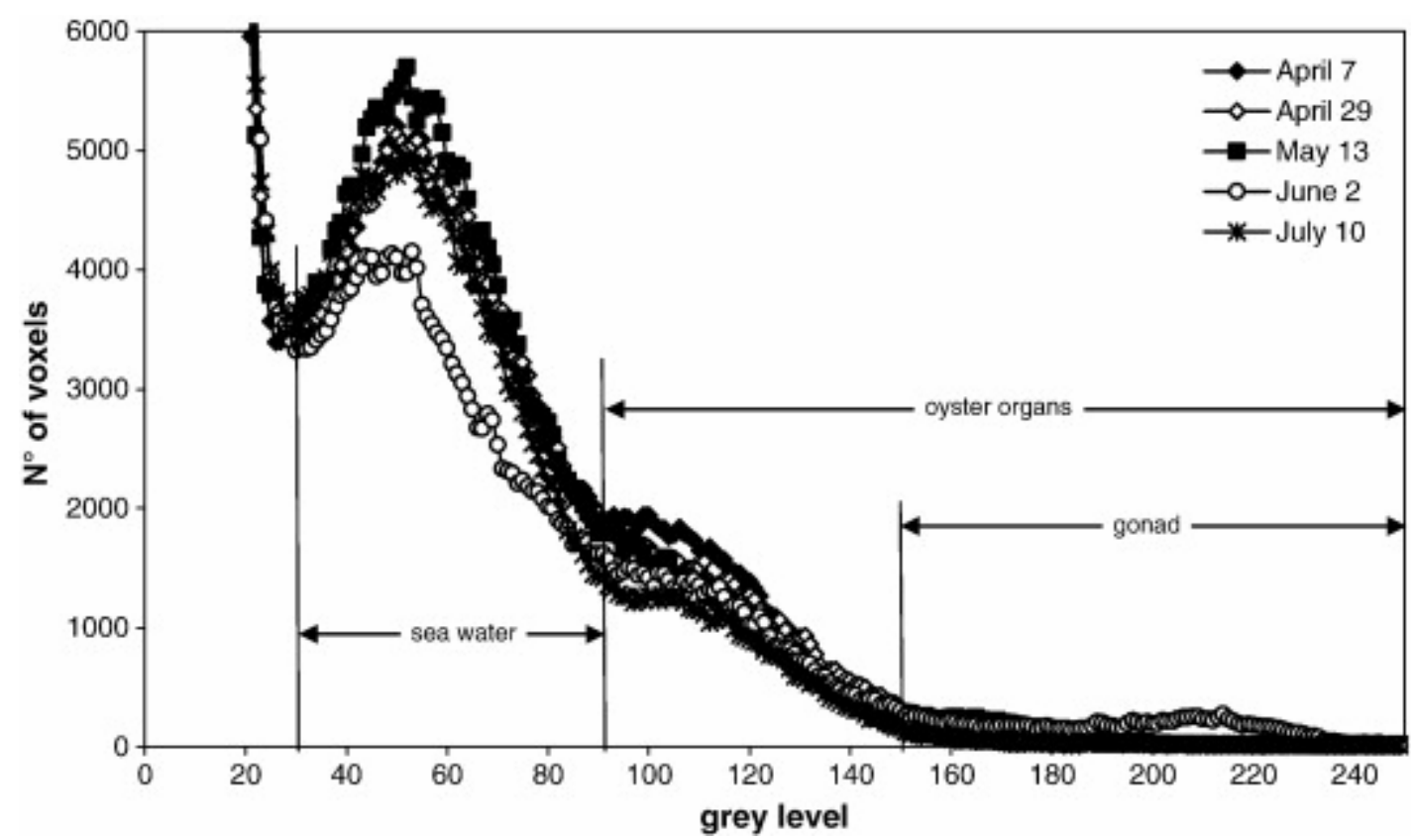

Fig. 2. Grey-level histograms from the 52 images from the T1-weighted Spoiled Gradient Echo for oyster 12 at the five dates of MRI scanning. 


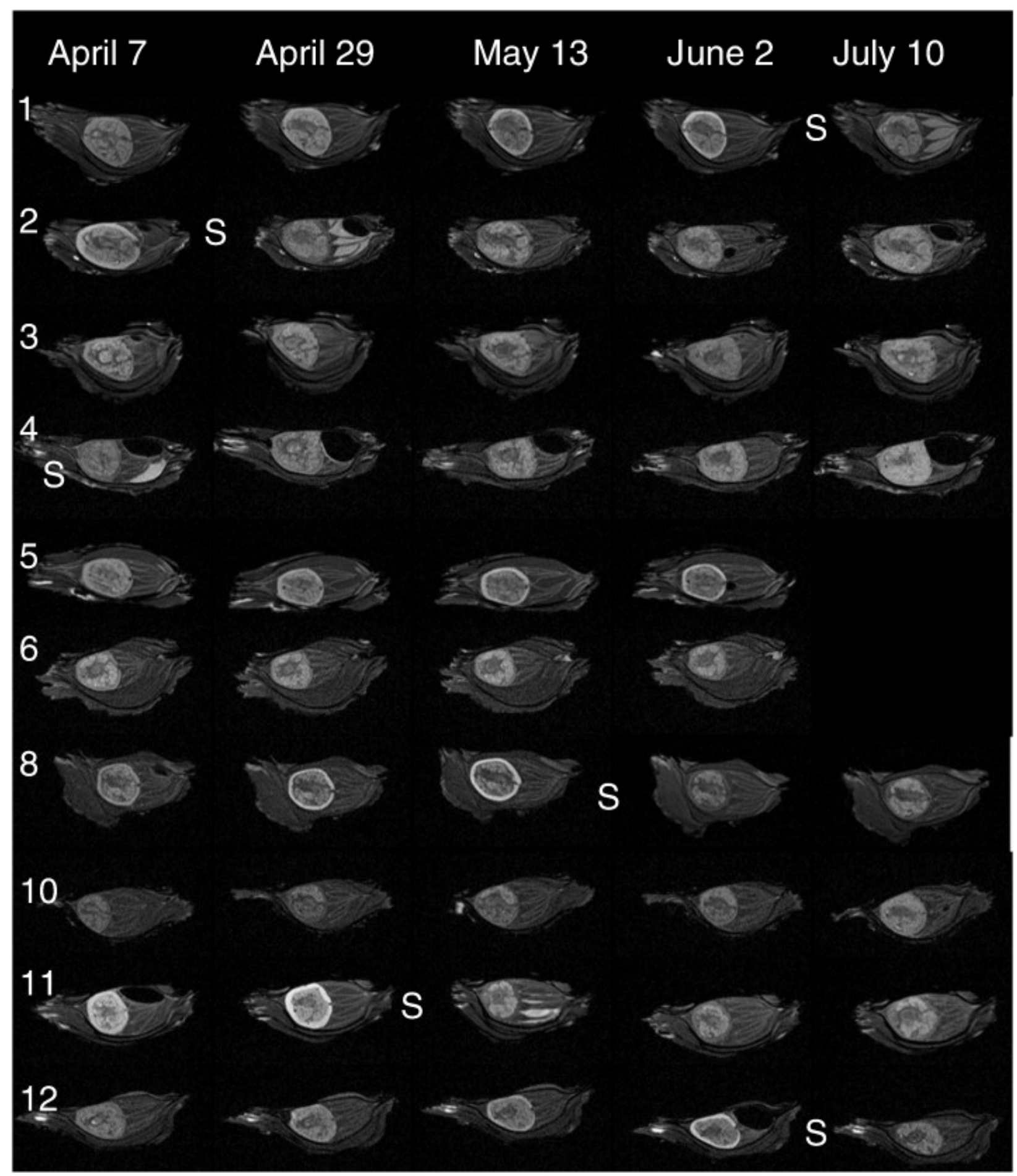

Fig. 3. The most representative MRI scanning images of gonad development of 10 laboratory conditioned flat oysters collected at five successive dates from April to July 2009 (S: spawning period) 


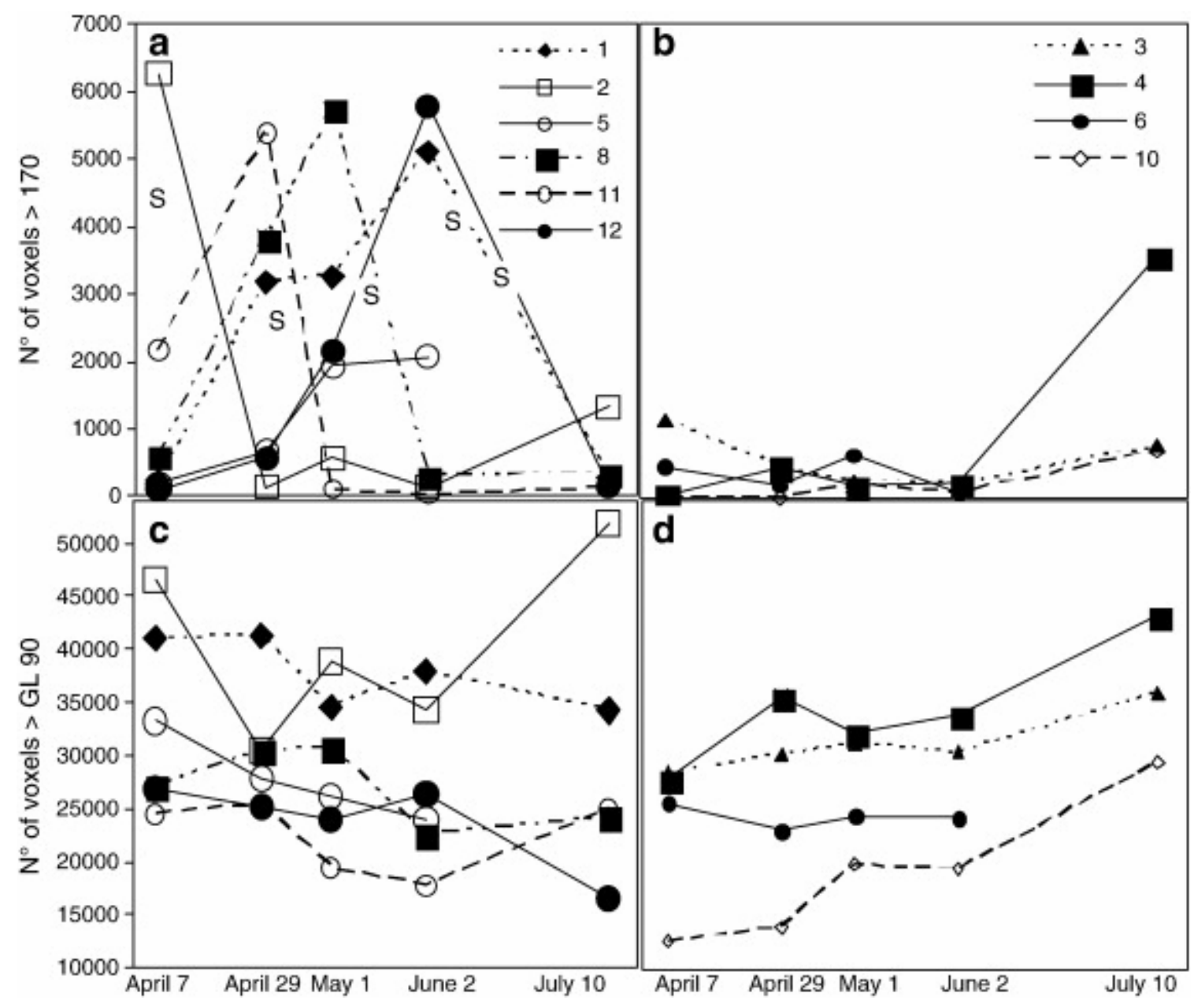

Fig. 4. Evolution of the gonad index $(a, b)$ and the somatic index (c, d) of 6 oysters with apparent development of the gonad $(a, c)$ and 4 oysters without apparent development of the gonad (b,d) at five dates of MRI scanning during April-July 2009. 